\title{
Anti-inflammatory properties of anesthetic agents
}

\author{
Fernanda Ferreira Cruz ${ }^{1}$, Patricia Rieken Macedo Rocco ${ }^{1}$ and Paolo Pelosi $2^{*^{*}}$
}

\begin{abstract}
This article is one of ten reviews selected from the Annual Update in Intensive Care and Emergency Medicine 2017. Other selected articles can be found online at http://ccforum.com/series/annualupdate2017. Further information about the Annual Update in Intensive Care and Emergency Medicine is available from http://www.springer.com/series/8901.
\end{abstract}

\section{Background}

For more than a century, experimental and clinical studies have reported that anesthetic agents have diverse effects on the immune system [1]. Despite rapid development in the fields of immunology and anesthesiology in recent decades, the specific mechanisms by which each anesthetic drug affects the immune system remain unclear. Here, we will define innate and adaptive immunity, present factors that can lead to immune dysregulation during the perioperative period, describe the effects of some of the most common anesthetic drugs on immune cells and cytokines, and discuss the possible clinical implications of the use of these drugs [2].

\section{Innate and adaptive immunity}

The immune system plays a vital role in survival by protecting us from the many potentially deadly infectious pathogens in our environment, as well as from cancer cells. The immune system is able to recognize pathogens and trigger their elimination through innate and then adaptive immune responses [3].

Innate immunity, also called natural or native immunity, is the first line of defense and refers to protective mechanisms that are present even before infection. Its principal components are the epithelial membranes (which block pathogen entry), phagocytic cells (neutrophils

\footnotetext{
* Correspondence: ppelosi@hotmail.com

${ }^{2}$ Department of Surgical Sciences and Integrated Diagnostics, IRCCS AOU

San Martino IST, University of Genoa, 16132 Genoa, Italy

Full list of author information is available at the end of the article
}

and macrophages), dendritic cells, natural killer (NK) cells and several plasma proteins, including the complement system. The most important cellular reaction of innate immunity is inflammation - the process, mediated by dendritic and NK cells, whereby phagocytic cells are recruited and activated to eliminate aggressor agents [2,3].

Adaptive immunity, also called specific or acquired immunity, consists of mechanisms that are induced by the recognition of specific pathogen antigens. The adaptive immune system is mediated primarily by lymphocytes, and its function can be classified into two types: humoral immunity, mediated by B-lymphocytes and their secreted antibodies; and cell-mediated or cellular immunity, mediated mostly by T-lymphocytes and their cytokines, which play an important role in immune cell activation, regulation, and communication $[2,3]$.

Besides its role in host defense against infectious agents and tumor cells, the inflammatory response is essential for tissue reconstitution after injury caused by accidental or surgical insults. Dysregulation of this inflammatory process may increase susceptibility to infections, accelerate the growth and metastasis of residual cancer cells, and result in postoperative complications, such as wound healing disturbances and infections leading to sepsis followed by multiple organ failure and death [4].

\section{Perioperative immunosuppression}

The perioperative immunosuppression observed in surgical patients is related to the neuroendocrine stress exerted through activation of the autonomic nervous system and the hypothalamic-pituitary-adrenal (HPA) axis. Surgical stress-induced release of hormones such as catecholamines (norepinephrine and epinephrine), adrenocorticotropic hormone and cortisol, via the autonomic nervous system and the HPA, mediates inhibitory effects on immune functions, as monocytes/macrophages and T-cells express both $\beta 2$-adrenoreceptors and glucocorticoid receptors, which promote cellular signaling to 
inhibit the production of proinflammatory cytokines [5]. Moreover, cytokines such as interleukin (IL)-1, IL-6, and tumor necrosis factor (TNF)- $\alpha$ from monocytes/ macrophages and lymphocytes activated by surgical stress may stimulate the HPA [6]. Therefore, the neuroendocrine system, as well as proinflammatory and anti-inflammatory cytokines, synergistically augments its suppressive effects on the immune system in the perioperative period.

In addition to the surgical stress response, intraoperative blood pressure management, blood transfusion, hyperglycemia, hypothermia, postoperative pain, and anesthesia, all of which are managed by anesthesiologists during surgical interventions, cause perioperative immunosuppression (Fig. 1; [4]). Anesthetic agents and anesthesia management are suspected of impairing several aspects of the inflammatory response process, either indirectly by modulating the stress response or directly by disturbing the functions of immune cells $[1,2,4,7]$.

Indeed, anesthetic drugs induce analgesia by affecting the transmission of nerve impulses and modulate surgical stress by acting on the HPA axis, thus affecting its immunomodulatory effects $([1,2,4]$; Fig. 1). Recently, numerous studies have shown that, alongside the immunosuppression caused by surgical stress, anesthetics and analgesic agents commonly used in surgery and in intensive care may directly affect the functions of immunocompetent cells $[4,8,9]$.

Diverse in vitro experiments with human immune cells ex vivo [2], in vivo tests [10] and animal models have demonstrated a wide range of effects of anesthetic drugs on the immune system, including changes in immune cell counts and their functionality and effects on the secretion patterns of diverse immune mediators, affecting the inflammatory response in the postoperative period [1-3].

\section{Effects of general anesthesia on inflammation}

The immune-modulating effects of anesthetics in vitro were first demonstrated more than 100 years ago [7]. The increasing knowledge of recent years is strongly related to developments in basic science and improvements in laboratory technique, e. g., cell separation and cell culture methods. It has been demonstrated that, at concentrations used clinically, different anesthetics depress the functions of the inflammatory response differentially.

\section{Ketamine}

Ketamine, an N-methyl-d-aspartate (NMDA)-receptor antagonist, acts at different levels of inflammation, interacting with inflammatory cell recruitment, cytokine production, and regulation of inflammatory mediators [11]. The immune-inhibitory effects of ketamine were recently found to be partly due to inhibition of transcription factor activator protein-1 and nuclear factorkappa B (NF-kB), which regulate the production of several proinflammatory mediators [12]. The notion that ketamine interferes with immunity comes from the early observations of improved outcomes in critically ill patients and in experimental septic shock [13]. In vivo, a subanesthetic dose of ketamine produced a dose-dependent decrease in mortality with a significant reduction in production of TNF- $\alpha$ and IL-6 in septic rats [11, 14]. Intravenous administration of ketamine abolished albumin extravasation in a rat model of chemical peritonitis [14]. In other studies, anesthetic doses of ketamine attenuated lipopolysaccharide (LPS)-induced liver injury, with a reduction in cyclooxygenase $(\mathrm{COX})-2$, inducible nitric oxide synthase (iNOS), and NF-kB-binding activity $[11,14]$. These data clearly indicate that ketamine may exert anti-inflammatory actions in vivo. These antiinflammatory effects have also been found in clinical settings. Low-dose ketamine $(0.25-0.5 \mathrm{mg} / \mathrm{kg})$ significantly

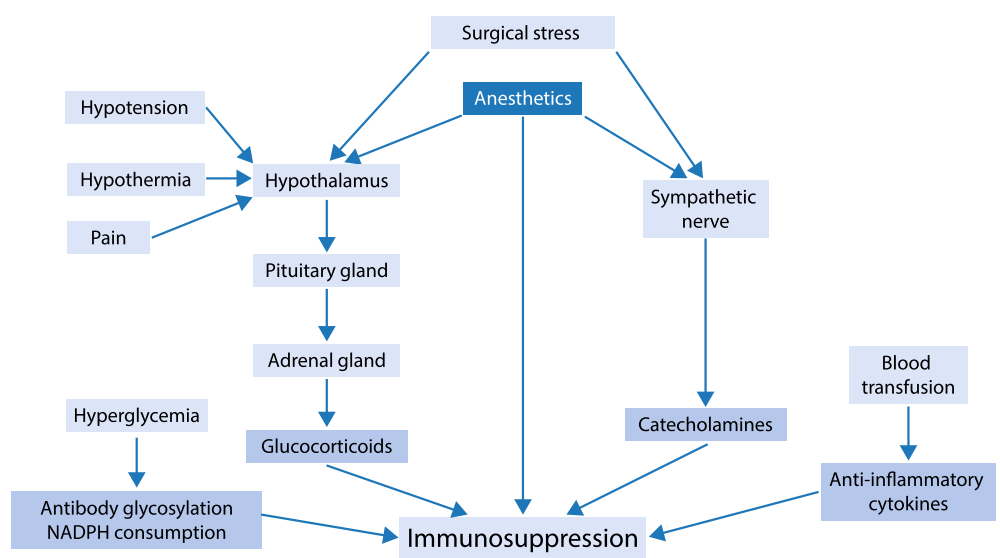

Fig. 1 Schematic diagram of possible modulators of immune competence during anesthesia and surgery. Anesthetics have direct effects on the immune system and indirect effects through neuro-immune-endocrine interactions during surgical stress 
suppressed intraoperative and postoperative increases in serum IL-6 and C-reactive protein (CRP) in patients undergoing coronary artery bypass graft (CABG) surgery with cardiopulmonary bypass $(\mathrm{CPB})[11,14]$ and significantly decreased superoxide production. However, low-dose ketamine was not shown to have any antiinflammatory effects in low-risk patients undergoing offpump CABG. The link remains controversial [14].

\section{Midazolam}

Midazolam, a widely used benzodiazepine derivative, acts on GABA receptors by increasing neuronal permeability to chloride ions, leading to cell hyperpolarization. It is known to inhibit certain aspects of immune function [15]. Midazolam binds to peripheral receptors on macrophages and modulates their metabolic oxidative responsiveness in vitro. It has been suggested that clonazepam also binds to receptors on macrophages and inhibits their capacity to produce IL-1, IL-6, and TNF- $\alpha$ in a T-cell independent manner [16]; however, it was ineffective. These results demonstrate an in vivo immunosuppressive property of peripheral and mixed benzodiazepine receptor agonists (midazolam and diazepam) but not central-type receptor agonists (clonazepam), affecting characteristic phagocyte functions involved in host-defense mechanisms as well as in the inflammatory response [15]. Midazolam is also able to inhibit human neutrophil function and the activation of mast cells induced by TNF- $\alpha$ in vitro, and suppresses expression of IL-6 mRNA in human blood mononuclear cells [17]. When administered to LPS-stimulated macrophages, midazolam suppressed the respiratory burst of reactive oxygen species (ROS), inhibited NF- $\mathrm{B}$ B activation via suppression of IкB- $\alpha$ degradation, and inhibited p38 activation, which has been reported to play a critical role in LPS-mediated COX-2 and iNOS expression, pathways involved in the proinflammatory macrophage phenotype [18]. Nonetheless, midazolam infusion did not affect cytokine production in septic patients [15].

\section{Propofol}

Propofol, another GABA receptor agonist, has been shown to impair several monocyte and neutrophil functions of the innate immune system, including respiratory burst [19], chemotaxis [20], phagocytosis [21] and polarization [4]. While some authors have showed that the inhibitory properties on human neutrophils and complement activation of propofol are related to its lipid carrier vehicle $[4,22]$, others have suggested that propofol at least partly inhibits human neutrophil chemotaxis by suppressing the $\mathrm{p} 44 / 42$ mitogen-activated protein kinase (MAPK) pathway [4, 20]. In clinically relevant concentrations, propofol inhibits the production of a chemotactic agent in human neutrophils $[4,20]$. The proliferative-suppressing effects of propofol were only observed in polymorphonuclear leukocytes (PMNs) obtained from critically ill patients who were primarily immunosuppressed [23]. Lymphocyte proliferation [4] and cytokine release in response to endotoxin were not found to be impaired in whole blood culture medium obtained from healthy volunteers [4, 24]. In an animal model of endotoxin-induced lung injury, propofol had anti-inflammatory effects. The underlying molecular mechanisms are still unclear; however, propofol is not known to inhibit activation of NF- $\kappa B$ [4]. Recent data suggest that propofol produces only cell-mediated immunomodulatory effects on innate immunity, and that these effects might be generated by its lipid solvent [4].

\section{Opioids}

The link between opioid use and alterations in host immune function is often mentioned in the literature, and has been formally documented since the early $19^{\text {th }}$ century. The increased incidence of various local and systemic infections in intravenous drug abusers led to the conclusion that the causative link between drug use and infections could not be simply explained by the injection process, but that opiates themselves were acting to modulate immune function [25]. Different opioids affect immune function differently depending on drug factors, host factors, and the duration of exposure [26]. Morphine, fentanyl, remifentanil, methadone and codeine present strong immunomodulatory effects, while tramadol, hydrocodone, oxycodone, and buprenorphine present much weaker or no immune-modulating capacity [25]. This feature of opioids is often linked to central neuro-endocrine/neuro-paracrine and peripheral mechanisms and to peripheral actions mediated by mu-opioid receptors on immune cells [25].

The importance of centrally mediated mechanisms is supported further by the observation that opioids that cross the blood brain barrier (BBB) exert more immunomodulatory effects than opioids that do not cross the BBB [27]. Although opioid effects are largely attributed to decreased central sympathetic nervous system outflow, opioids can also cause direct sympathetic nervous activation, which may suppress the proliferation and function of some immune cell populations and primary and secondary lymphoid tissues [28]. The interaction of opioids with the HPA axis and its components (ACTH and cortisol production) is complex, species- and timedependent, with different effects after acute and chronic administration. In humans, data are scarce; however, current evidence suggests that acute administration of opioids results in either a reduction or no change in $\mathrm{ACTH}$ or glucocorticoids. There is evidence that opioids attenuate the circadian rhythm of ACTH and cortisol, leading to consistent increments in circulating levels of 
these hormones, which might be sufficient to produce immune suppression [25].

Several studies have suggested that mu-opioid receptors are expressed on peripheral blood mononuclear cells $[4,29,30]$; however, in contrast to previous reports and despite using several validated methodologies, a recent investigation was unable to detect any opioid receptors or transcripts in mononuclear cells collected from venous blood [25].

There are well-documented, dose-dependent, immunosuppressive effects of morphine, which is known to impair monocyte and neutrophil function, NK cell-mediated cytotoxicity, lymphocyte and macrophage proliferation and cytokine release. Morphine promotes apoptosis by direct activation of the enzymes involved in cell apoptosis, inhibits leukocyte function by increasing intracellular concentrations of NO and cyclic AMP, and by inhibiting nuclear NF- $\mathrm{kB}$ via NO-dependent mechanisms [4]. Recent studies of the effects of synthetic opioids used in general anesthesia showed no more than transient immunomodulatory changes $[1,2,4]$.

Fentanyl is known to enhance NK-cell cytotoxicity and increase NK and cytotoxic (CD8+) cell counts; however, the production of superoxide by PMNs and the number of circulating B- and T-lymphocytes remained unchanged in healthy volunteers $[4,31]$. These effects of fentanyl on NK cells seem to be more centrally mediated, as fentanyl does not affect NK-cell activity directly. In two studies, sufentanil and alfentanil were observed to produce inhibitory effects on leukocyte migration, NK-cell activity, and mitogen-induced lymphocyte proliferation [1, 2, 4, 32].

\section{Thiopental}

When administered over prolonged periods, short- and intermediate-acting barbiturates, which act as GABA receptor agonists, may induce iatrogenic immunosuppression. A higher incidence of infections has been described in head-injured patients with increased intracranial pressure (ICP) who received prolonged infusions of thiopental $[4,33]$. Thiopental is one of the most investigated anesthetic agents and is widely used for the induction of general anesthesia. Its inhibitory effects on the nonspecific immune system have been well documented in several studies. In clinically used concentrations, thiopental has been shown to inhibit the bactericidal functions of leukocytes; neutrophil polarization, chemotaxis, adherence, phagocytosis, and respiratory burst; and monocyte chemotaxis [2]. In high concentrations, thiopental affects neutrophil and monocyte phagocytosis. In short, the described inhibitory effects of thiopental are indicative of direct cell-mediated inhibition of the immune response and a strong anti-inflammatory effect. In addition, thiopental is known to depress mitogen/ antigen-induced lymphocyte proliferation in different culture mediums, and decreases the quantity of cytokines released in response to mitogens or endotoxins [34]. Recent studies have suggested that thiopental inhibits NF- $\mathrm{kB}$ activation [4]. Clinically, the immunosuppressive effects of thiopental are probably of minor clinical relevance, since it is often used only for induction of anesthesia [20].

\section{Dexmedetomidine}

Dexmedetomidine, an agonist of $\alpha_{2}$-adrenergic receptors in certain regions of the brain, has been shown to reduce proinflammatory cytokine levels in experimental sepsis [35] as well as in critically ill $[1,4]$ and postoperative patients [36]. A significant decrease in leukocyte counts, CRP, IL-6, IL- 8 and TNF- $\alpha$ levels in dexmedetomidinetreated patients is indicative of its anti-inflammatory potential when used as a perioperative adjunct $[1,2,4]$. A number of mechanisms of action have been postulated for dexmedetomidine, including: modulation of cytokine production by macrophages/monocytes during the stress response, which may also be stimulated via $\alpha 2$ adrenoceptors; inhibition of apoptosis; central sympatholytic effects, including stimulation of cholinergic anti-inflammatory pathways; and antinociceptive action involving interactions between pain and immune factors (proinflammatory cytokines) [1, 2, 4]. So far, however, these mechanisms remain unclear [37].

\section{Volatile anesthetics}

Inhalational anesthetic agents have inhibitory effects on neutrophil function, decrease lymphocyte proliferation, and suppress cytokine release from peripheral blood mononuclear cells $[1,2,38]$. Halogenated anesthetics are known to suppress inflammatory cytokines in rat alveolar cells [4]. In contrast, exposure to volatile anesthetics and mechanical ventilation has been shown to induce increased gene expression of proinflammatory cytokines [38]. Volatile anesthetics affect the expression of iNOS by reversible inhibition of voltage-dependent calcium channels and decreased intracellular calcium concentrations. Thus, the in vitro effects of volatile anesthetics predominantly consist of inhibition of immune products, but these are generally transient, as well as dose- and time-dependent $[1,4,38]$.

\section{Sevoflurane}

Anesthetic preconditioning to sevoflurane has been shown to promote protection from endotoxemia, ischemia-reperfusion injury, myocardial ischemiareperfusion injury, among other disease models. Sevoflurane attenuated the activation of NF- $\mathrm{kB}$ and subsequent expression of NF-kB-dependent inflammatory mediators via Toll-like receptors (TLRs) [38]. Additionally, sevoflurane 
protects against vascular endothelium dysfunction induced by oxidative stress and inflammation through activation of the eNOS/NO pathway and inhibition of NF- $\mathrm{kB}$. Endothelial dysfunction induced by oxidative stress and inflammation plays a critical role in the pathogenesis of cardiovascular diseases [37]. In particular, sevoflurane has been shown to induce a more pronounced suppression of cytokine release than isoflurane or enflurane.

\section{Isoflurane}

Isoflurane exposure leads to reduction in leukocyte counts and levels of systemic proinflammatory cytokines (TNF- $\alpha$, IL-6 and IL-1 $\beta$ ), as well as less macrophage activation and polarization toward the M2 phenotype. These effects were found to be protein kinase Cdependent and also due to systemic inhibition of NF- $\mathrm{KB}$ [38]. These findings suggest that pre-exposure to volatile anesthetics induces a systemic anti-inflammatory effect. On the other hand, exposure to isoflurane has been shown to lead to cognitive impairment and a small increase in IL-1 $\beta$ and activated caspase- 3 levels in the hippocampi of both young adult and elderly rats. These results suggest that isoflurane induces neuroinflammation, which then leads to cognitive impairment.

Little is known regarding the mechanisms of volatile anesthetic-induced neuroinflammation, but isoflurane has been shown to open the $\mathrm{BBB}$, increasing the permeation of intravascular substances into brain tissue. A recent study showed that exposure of $\mathrm{H} 4$ human neuroglioma cells to $2 \%$ isoflurane for $6 \mathrm{~h}$ activated NF- $\mathrm{kB}$, increasing inflammatory cytokine production. Therefore, local activation of NF- $\mathrm{kB}$ is presumably a mechanism for isofluraneinduced neuroinflammation [39].

\section{Effects of regional and local anesthesia on inflammation}

With regard to their anti-inflammatory properties, local anesthetics have been shown to affect PMNs directly, as well as macrophage and monocyte function. Ropivacaine and lidocaine (100-300 mM) decreased TNF- $\alpha$-induced upregulation of $\mathrm{CD} 11 \mathrm{~b} / \mathrm{CD} 18$ surface expression on PMNs in vitro [40]. Thus, local anesthetics decrease PMN adherence, migration, and accumulation at the site of inflammation.

Since local anesthetics impair PMN presence and function, concerns have arisen that local anesthesia might increase susceptibility to infection, as local anesthetic-mediated depression of the PMN oxidative metabolic response may decrease the host's ability to control bacterial proliferation [41]. Antibacterial effects of local anesthetics have been reported in vitro and in vivo, but only at millimolar concentrations. Lidocaine (37 mM), for example, inhibits the growth of Escherichia coli and Streptococcus pneumoniae, but has no effect on
Staphylococcus aureus or Pseudomonas aeruginosa. In contrast, other authors found that lidocaine reduced growth of all of the above-mentioned bacteria. Using a guinea pig wound model, lidocaine $(74 \mathrm{mM})$ induced reduction of bacterial growth to approximately $30 \%$ in $S$. aureus-contaminated wounds. In summary, the antiinflammatory properties of local anesthetics at systemic concentrations might, theoretically, increase the risk of infection, since antibacterial and antiviral effects are only attained with the use of high concentrations [1-3, 40, 41]. Nevertheless, this seems to be of minor importance in most studies, except for settings of severe bacterial contamination. Local anesthetics are known for their inhibition of excessive inflammatory responses without significant impairment of host immunity.

\section{Clinical implications of the anti-inflammatory properties of anesthetics}

Investigations of the immune effects of anesthetics have been derived mostly from in vitro studies, because human clinical studies are more complex, involving such variables as type and duration of surgery and patient complications. Although it is difficult to distinguish the relative contributions of surgical stress, anesthetics and analgesic agents to the patient's immune system, anesthesiologists must not ignore the immunosuppressive effects of anesthetic drugs on perioperative immunity [1].

\section{In the perioperative period}

Surgical trauma induces an endocrine, metabolic, hemodynamic and immune response that persists for at least a few days. It is generally accepted that the effect of anesthetics on the immune system is modest compared to the effects induced by major surgery or trauma; thus, anesthetics may not have any clinically significant effects on immune function in healthy patients anesthetized for short procedures, in whom the inflammatory response is usually balanced, well controlled and of limited duration [42]. An immunosuppressive effect of approximately $20 \%$ might not have great consequences for an immunocompetent patient. However, if the patient has a genetic predisposition to immune impairment or is already compromised, e. g., by aging, tumor burden, diabetes mellitus or malnutrition, the immunosuppressive effects of anesthetics might play a salient role in postoperative infectious complications, morbidity and mortality [43]. In addition, an increasing number of immunosuppressed elderly patients require anesthesia and intensive care treatment. Thus, the possible immunomodulatory effects of anesthetics must be widely comprehended and agents chosen wisely. Particularly in patients with cancer, immunosuppression after surgery accelerates the growth of residual malignant cells and promotes the establishment of new metastases $[1,42,44]$. On the other hand, 
the immunosuppressive effects of anesthetics that lead to anti-inflammatory responses may be therapeutically beneficial in distinct situations such as ischemia-reperfusion injury, the systemic inflammatory response syndrome, or acute respiratory distress syndrome (ARDS) [45]. Therefore, the effects of anesthetics on immunity may be not only adverse but also beneficial on the prognosis of specific patients. The immune effects of surgery and anesthetics affect the long-term outcomes of patients after surgery. Therefore, awareness of these immunological properties is helpful for daily anesthetic management.

In brief, the effect of anesthetics on the immune system has been less investigated in vivo than in vitro; current findings are contradictory and indicative of only minor clinical importance. At present, no one mode of anesthesia can be recommended in favor of another in terms of effect on the inflammatory response $[1,2,4]$.

In addition to effects on non-specific cell-mediated immunity, some local anesthesia techniques can inhibit parts of the neuroendocrine response to surgery. Subarachnoid and epidural blockade blunts the increase in plasma levels of epinephrine, norepinephrine and cortisol induced by surgery. The reduction in the neuroendocrine stress response is most pronounced in connection with surgical procedures on the lower abdomen and lower extremities. In these cases, epidural anesthesia can provide complete blockade of all afferent neurogenic stimuli from the surgical field [40, 41], reducing the stress-related response, and can even reduce perioperative mortality and postoperative morbidity after some surgical procedures $[1-4,40,41]$.

\section{In the intensive care unit}

While a slight immunosuppressive effect of anesthesia during surgery is probably of minor importance because of the limited duration of exposure, critical care settings pose a different set of challenges. In the ICU, patients are often exposed to anesthetic agents for several days, and the side effects of immunosuppression have been shown to be clinically important in this population. In this context, as early as 1956, bone-marrow depression was described after prolonged ventilation with nitrous oxide [46].

Later, in 1983, increases in ICU mortality were reported after the introduction of etomidate for sedation [47]. Patients sedated with etomidate had a mortality of $77 \%$, versus only $28 \%$ in patients not given this agent. It was later discovered that etomidate inhibits the synthesis of cortisol precursors and thus reduces levels of plasma cortisol. A high rate of infections has also been described in ICU patients receiving long-term thiopental infusions.
Propofol or benzodiazepines are most often used for long-term sedation in the ICU. Animal studies have shown reduced defense against infection following longterm infusion of propofol as well as benzodiazepines. A single study has shown increased plasma levels of proinflammatory cytokines (IL-1, IL-6, TNF- $\alpha$ ) following long-term propofol infusion, while a reduction in proinflammatory cytokines was observed following benzodiazepine infusion. To avoid a potential detrimental effect of long-term sedation in critically ill patients, the duration and the doses of sedative agents should be kept as low as possible, not least because these patients are immunocompromised. Daily wake-up trials are recommended to reduce the risk of oversedation [1-4].

\section{Conclusion}

Anesthetics have long been suspected of impairing various aspects of immune system function, either indirectly by modulating the stress response or directly by affecting the functioning of immunocompetent cells. Although these effects are transient and may be of minor importance in subjects with normal immune systems, in patients with pre-existing immune dysfunction or multiple organ failure and in other high-risk groups, the influence of anesthetics and of the choice of anesthetic management technique on the perioperative inflammatory response may have clinical implications.

The effects of anesthetics on immunomodulation of inflammation are complex; immunosuppression can have positive as well as negative impact. Therefore, the choice and use of anesthetic agents are highly dependent on the immune status of each patient. Possible hazards associated with perioperative immunosuppression include increased risk of tumor metastasis and infection, whereas the anti-inflammatory effects of anesthetics may instead provide benefit in conditions associated with systemic and local inflammation [4].

\section{Acknowledgements}

Not applicable.

\section{Funding}

Publication costs were funded by the Department of Surgical Sciences and Integrated Diagnostics (DISC), University of Genova, Genova, Italy. This study was supported by Conselho Nacional de Desenvolvimento Científico e Tecnológico/Ministério da Saúde/DECIT (469716/2014-2, 465064/2014-0, and 400462/2014-1 to PRMR) and Fundação Carlos Chagas Filho de Amparo à

Pesquisa do Estado do Rio de Janeiro (E-26/103.118/2 to PRMR).

Availability of data and materials Not applicable.

Authors' contributions

CRUZ, PRMR, PP contributed to the literature review and the drafting of the manuscript. All authors read and approved the final manuscript.

Competing interests

The authors declare that they have no competing interests. 


\section{Consent for publication}

Not applicable.

\section{Ethics approval and consent to participate}

Not applicable.

\section{Author details}

${ }^{1}$ Federal University of Rio de Janeiro, Laboratory of Pulmonary Investigation Carlos Chagas Filho Institute of Biophysics, 21941-902 Rio de Janeiro, Brazil. ${ }^{2}$ Department of Surgical Sciences and Integrated Diagnostics, IRCCS AOU San Martino IST, University of Genoa, 16132 Genoa, Italy.

\section{Published online: 21 March 2017}

\section{References}

1. Kurosawa S, Kato M. Anesthetics, immune cells, and immune responses. J Anesth. 2008;22:263-77.

2. Colucci DG, Puig NR, Hernandez PR. Influence of anesthetic drugs on immune response: from inflammation to immunosuppression. OA Anesthetics. 2013;1:21-38.

3. Friedman H, Newton C, Klein TW. Microbial Infections, Immunomodulation, and Drugs of Abuse. Clin Microbiol Rev. 2003;16(2):209-19.

4. Schneemilch CE, Schilling T, Bank U. Effects of general anaesthesia on inflammation. Best Pract Res Clin Anaesthesiol. 2004;18:493-507.

5. Elenkov IJ, Chrousos GP. Stress hormones, proinflammtory and anti-inflammatory cytokines, and autoimmunity. Ann NY Acad Sci. 2002:966:290-303.

6. Kennedy BC, Hall GM. Neuroendocrine and inflammatory aspects of surgery: do they affect outcome? Acta Anaesthesiol Belg. 1999;50:205-9.

7. Graham EA. The influence of ether and ether anesthesia on bacteriolysis, agglutination and phagocytosis. J Infect Dis. 1911;8:147-75.

8. Amin OAl, Salah HE. The effect of general or spinal anaesthesia on pro- and anti-inflammatory intracellular cytokines in patients undergoing appendicectomy using flow cytometric method. Egypt J Anaesth. 2011;27:121-5

9. Colucci D, Harvey G, Gayol MC, Elena G, Puig N. Halothane anesthesia in mice: effect on the phagocytic activity and respiratory burst of peritoneal macrophages. Neuroimmunomodulation. 2011;18:11-18.

10. Simeonova GP, Slovov E, Usunov R, Halacheva K, Dinev DN. Increased apoptosis of peripheral blood mononuclear cells (PBMC) during general and epidural anaesthesia in dogs. Vet Res Commun. 2008;32:619-26.

11. Loix S, De Kock M, Henin P. The anti-inflammatory effects of ketamine: state of the art. Acta Anaesthesiol Belg. 2011;62:47-58

12. Welters ID, Hafer $G$, Menzebach A, et al. Ketamine inhibits transcription factors activator protein 1 and nuclear factor-kappab, Interleukin-8 production, as well as CD11b and CD16 expression: studies in human leukocytes and leukocytic cell lines. Anesth Analg. 2010;110:934-41.

13. Kock M, Loix S, Lavand'homme P. Ketamine and peripheral inflammation. CNS Neurosci Ther. 2013;19:403-10.

14. Hirota K, Lambert DG. Ketamine: new uses for an old drug? $\mathrm{Br} J$ Anaesth. 2011;107:123-6

15. Memiş D, Hekimoğlu S, Vatan I, Yandım T, Yüksel M. Effects of midazolam and dexmedetomidine on inflammatory responses and gastric intramucosal pH to sepsis, in critically ill patients. Br J Anaesth. 2007:98:550-2.

16. Zavala F, Haumont J, Lenfant M. Interaction of benzodiazepines with mouse macrophages. Eur J Pharmacol. 1984;106:561-6.

17. Nishina K, Akamatsu H, Mikawa K, et al. The inhibitory effects of thiopental, midazolam, and ketamine on human neutrophil functions. Anesth Analg. 1998;86:159-65.

18. Kim SN, Son SC, Lee SM, et al. Midazolam inhibits proinflammatory mediators in the lipopolysaccharide-activated macrophage. Anesthesiology. 2006:105:105-10.

19. Frohlich D, Rothe G, Schwall B, et al. Thiopentone and propofol, but not methohexitone nor midazolam, inhibit neutrophil oxidative responses to the bacterial peptide FMLP. Eur J Anaesthesiol. 1996:13:582-8.

20. Jensen $A G$, Dahlgren C, Eintrei C. Propofol decreases random and chemotactic stimulated locomotion of human neutrophils in vitro. Br J Anaesth. 1993;70:99-100.

21. Mikawa K, Akamatsu H, Nishina K, et al. Propofol inhibits human neutrophil functions. Anesth Analg. 1998;87:695-700.

22. Cleary TG, Pickering LK. Mechanisms of intralipid effect on polymorphonuclear leukocytes. J Clin Lab Immunol. 1983;11:21-6.
23. Pirttinkangas CO, Perttila J, Salo M. Propofol emulsion reduces proliferative responses of lymphocytes from intensive care patients. Intensive Care Med. 1993;19:299-302.

24. Hoff G, Bauer I, Larsen B, Bauer M. Modulation of endotoxin-stimulated TNF-alpha gene expression by ketamine and propofol in cultured human whole blood. Anaesthesist. 2001;50:494-9.

25. Al-Hashimi M, Scott WM, Thompson JP, Lambert DG. Editor's choice: Opioids and immune modulation: more questions than answers. $\mathrm{Br} J$ Anaesth. 2013:111:80-8.

26. Sacerdote P. Opioids and the immune system. Palliat Med. 2006;20:s9-s15.

27. Hernandez M, Flores L, Bayer B. Immunosuppression by morphine is mediated by central pathways. J Pharmacol Exp Ther. 1993;267:1336-41.

28. Hall DM, Suo $J$, Weber RJ. Opioid mediated effects on the immune system: sympathetic nervous system involvement. J Neuroimmunol. 1998;83:29-35.

29. Bidlack JM. Detection and function of opioid receptors on cells from the immune system. Clin Diagn Lab Immunol. 2000;7:719-23.

30. Rittner HL, Brack A, Machelska H, et al. Opioid peptide-expressing leukocytes: identification, recruitment, and simultaneously increasing inhibition of inflammatory pain. Anesthesiology. 2001;95:500-8.

31. Jacobs $R$, Karst $M$, Scheinichen $D$, et al. Effects of fentanyl on cellular immune functions in man. Int J Immunopharmacol. 1999;21:445-54.

32. Sacerdote P, Gaspani L, Rossoni G, et al. Effect of the opioid remifentanil on cellular immune response in the rat. Int J Immunopharmacol. 2001;1:713-9.

33. Eberhardt KE, Thimm BM, Spring A, Maskos WR. Dose-dependent rate of nosocomial pulmonary infection in mechanically ventilated patients with brain oedema receiving barbiturates: a prospective case study. Infection. 1992;20:12-8.

34. Chanimov M, Berman S, Weissgarten J, et al. Substances used for local and general anaesthesia in major surgery suppress proliferative responsiveness of normal rat peripheral blood mononuclear cells in culture. Eur J Anaesthes. 2000:17:248-55.

35. Cavalcanti V, Santos CL, Samary CS, et al. Effects of short-term propofol and dexmedetomidine on pulmonary morphofunction and biological markers in experimental mild acute lung injury. Respir Physiol Neurobiol. 2014:203:45-50.

36. Kang SH, Kim YS, Hong TH, et al. Effects of dexmedetomidine on inflammatory responses in patients undergoing laparoscopic cholecystectomy. Acta Anaesthesiol Scand. 2013;57:480-7.

37. Li B, Li Y, Tian S, et al. Anti-inflammatory effects of perioperative dexmedetomidine administered as an adjunct to general anesthesia: a meta-analysis. Sci Rep. 2015;5:12342.

38. Yoon-Mi L, Byeng CS, Kyung-Jin Y. Impact of volatile anesthetics on oxidative stress and inflammation. Biomed Res Int. 2015;242709.

39. Blum FE, Zuo Z. Volatile anesthetics-induced neuroinflammatory and anti-inflammatory responses. Med Gas Res. 2013;3:16.

40. Cassuto J, Sinclair R, Bonderovic M. Anti-inflammatory properties of loca anesthetics and their present and potential clinical implications. Acta Anaesthesiol Scand. 2006:50:265-82.

41. Hahnenkamp K, Herroeder S, Hollmann MW. Regional anaesthesia, local anaesthetics and the surgical stress response. Best Pract Res Clin Anaesthesiol. 2004;18:509-27.

42. Toft $P$, Tønnesen $E$. The systemic inflammatory response to anaesthesia and surgery. Curr Anaesth Crit Care. 2008;19:349-53.

43. Homburger JA, Meiler SE. Anesthesia drugs, immunity, and long-term outcome. Curr Opin Anaesthesiol. 2006:19:423-8.

44. Vallejo R, Hord ED, Barna SA, Santiago-Palma J, Ahmed S. Perioperative immunosuppression in cancer patients. J Environ Pathol Toxicol Oncol. 2003;22:139-46

45. Kelbel I, Weiss M. Anesthetics and immune function. Curr Opin Anaesthesiol. 2001;14:685-91.

46. Lassen HC, Henriksen E, Neukirch F, Kristensen HS. Treatment of tetanus; severe bone-marrow depression after prolonged nitrous-oxide anaesthesia. Lancet. 1956;270:527-30.

47. Fellows IW, Byrne AJ, Allison SP. Adrenocortical suppression with etomidate. Lancet. 1983;2:54-5. 